\title{
La autoestima en adolescentes agresores y víctimas en la escuela: La perspectiva multidimensional ${ }^{1}$
}

\section{Self-esteem in aggressive and victimized adolescents at school: The multidimensional perspective}

\section{Estefania ESTÉVEZ LÓPEZ* Belén MARTÍNEZ FERRER* Gonzalo MUSITU OCHOA*}

\section{RESUMEN}

Este estudio tiene como objetivo analizar las diferencias en autoestima entre adolescentes con problemas de agresividad en la escuela, adolescentes victimizados por sus iguales y adolescentes que son al mismo tiempo agresores y víctimas en el contexto escolar. Se adopta una concepción multidimensional de la autoestima y se analizan las dimensiones familiar, escolar, social y emocional. Para ello, participaron en la investigación 965 adolescentes con edades comprendidas entre los 11 y 16 años y escolarizados en cuatro centros de enseñanza de la Comunidad Valenciana. Los resultados obtenidos vienen a confirmar la existencia de diferencias entre agresores, víctimas y agresores/víctimas en la siguiente dirección: el grupo de agresores presenta las puntuaciones más elevadas en las dimensiones de autoestima social y emocional; el grupo de víctimas muestra los mayores niveles de autoestima familiar y escolar; finalmente, el grupo de agresores/víctimas es el que presenta, en general, las puntuaciones más bajas en las cuatro dimensiones de autoestima analizadas en el estudio.

\footnotetext{
* Universitat de València.

${ }^{1}$ Este artículo se ha elaborado en el marco del proyecto de investigación SEJ2004-01742 "Violencia en la escuela e integración escolar: Aplicación y evaluación de un programa de intervención", subvencionado por el Ministerio de Educación y Ciencia de España.
} 
La autoestima en adolescentes agresores y víctimas en la escuela: La perspectiva multidimensional

\title{
PALABRAS CLAVE
}

Autoestima, Escuela, Violencia, Victimización, Agresor, Victima.

\begin{abstract}
This study aims to analyze differences in self-esteem among aggressive adolescents at school, victimized adolescents by peers, and adolescents who are both aggressors and victims in the school context. This research adopts a multidimensional conception of selfesteem: family, school, social and emotional dimensions are analyzed. The sample was composed of 965 adolescents whose ages range from 11 to 16 years old and studying in four school of the Valencian Community. The results obtained confirm the existence of differences among aggressors, victims and aggressors/victims in the following way: the group of aggressors showed the highest scores in the dimensions of social and emotional self-esteem; the group of victims obtained the highest levels of family and school selfesteem; finally, the group of aggressors/victims showed, in general, the lowest scores in the four dimensions of self-esteem analyzed in the study.
\end{abstract}

\section{KEY WORDS}

Self-esteem, School, Violence, Victimization, Aggressor, Victim. 
En la literatura científica sobre violencia escolar se ha constatado en numerosas ocasiones la estrecha relación existente entre los problemas de victimización y la baja autoestima de las víctimas (Austin y Joseph, 1996; Guterman, Hahm y Cameron, 2002; Olweus, 1998; Musitu, Buelga, Lila y Cava, 2001). Sin embargo, los resultados relativos a la asociación entre la conducta agresiva y la autoestima son mucho más contradictorios. Algunos autores sostienen que los adolescentes agresivos presentan una autoestima más baja que aquellos sin problemas de conducta (Mynard y Joseph, 1997; O’Moore, 1997), mientras otros afirman que los agresores por regla general se valoran positivamente a sî mismos y muestran un nivel de autoestima medio o incluso alto (Olweus, 1998; Rigby y Slee, 1992).

Esta aparente contradicción de resultados se podría atribuir al tipo de instrumentos utilizados para obtener medidas de autoestima, y en concreto, a si el instrumento seleccionado proporciona una medida de autoestima global o bien multidimensional. Parece ser que las medidas de autoestima global, como por ejemplo la Escala de Autoestima de Rosenberg (1986), no reflejan posibles diferencias existentes entre agresores y victimas (Dorothy y Jerry, 2003; Rigby y Slee, 1992), mientras que cuando se analiza la autoestima desde un punto de vista multidimensional, agresores y victimas muestran un perfil diferente en algunos dominios (Andreou, 2000; O'Moore y Hillery, 1991).

Debemos tener en cuenta que la autoestima alude a la valoración que la persona hace de sí misma, de modo que por un lado, el adolescente puede tener una imagen general de sí mismo favorable 0 desfavorable, y por otro lado, puesto que se desenvuelve en diversos contextos como el familiar, el escolar y el social, también desarrolla una imagen de sí mismo específica en cada uno de ellos (Cava, Musitu y Vera, 2000). La autoestima, por tanto, refleja una actitud general o global hacia uno mismo, así como actitudes hacia aspectos específicos que no son equivalentes ni intercambiables (Rosenberg, Schooler, Schoenbach, Rosenberg, 1995). En este sentido, por ejemplo, un adolescente puede tener un buen concepto de sí mismo en el ámbito familiar, pero no en el académico, o viceversa (Cava y Musitu, 2003). Por ello, para comprobar si existen diferencias en autoestima entre agresores y victimas, es necesario adoptar una perspectiva multidimensional de este constructo (Herrero, Musitu y Gracia, 1995).

O'Moore y Kirkman (2001) utilizaron en su estudio medidas tanto globales como multidimensionales de autoestima para comprobar las diferencias entre agresores, víctimas y un tercer grupo de adolescentes que eran a la vez agresores/víctimas. Estos autores concluyen que tanto los agresores como las victimas presentan el mismo nivel de autoestima global, que además, es significativamente inferior al de aquellos adolescentes no implicados en problemas de agresión ni de victimización en la escuela; por otro lado, el grupo de agresores/victimas es el que obtuvo los niveles más bajos de autoestima global en comparación con el resto de grupos. Sin embargo, al analizar las distintas dimensiones de autoestima, los resultados mostraron que las víctimas se valoraban más positivamente en el dominio escolar, mientras que los agresores lo hacian en el dominio social. De hecho, no se encontraron diferencias significativas en autoestima social entre el grupo de agresores y el de adolescentes no implicados en problemas de violencia escolar.

También en el estudio de Andreou (2000), los adolescentes agresivos y 
aquellos sin problemas de agresión ni victimización en la escuela presentaron los niveles más elevados de autoestima social, las víctimas se situaron en una posición intermedia y las puntuaciones más bajas correspondieron al grupo de agresores/víctimas. En la dimensión de autoestima escolar, las víctimas ocuparon de nuevo la posición intermedia, aunque en esta ocasión tanto los agresores como los agresores/víctimas se valoraron más negativamente, mientras que las autopercepciones más positivas en el dominio escolar correspondieron a los adolescentes sin problemas de victimización ni agresión en la escuela.

Estos estudios se centran fundamentalmente en los dominios social y escolar de la autoestima pero no consideran, sin embargo, otras dimensiones como la familiar o la emocional. Estas dos dimensiones, la familiar y la emocional, han quedado relegadas en las investigaciones sobre violencia escolar, hasta el punto de que apenas existen referentes de su estudio en agresores y víctimas. No obstante, los resultados de algunos trabajos sobre el funcionamiento familiar 0 el bienestar emocional de agresores y víctimas, nos sugieren que también pueden existir diferencias entre estos adolescentes en ambas dimensiones. Por ejemplo, se ha constatado que las familias con hijos agresivos se caracterizan por la existencia de problemas de comunicación, frecuentes conflictos y falta de apoyo parental (Dekovic, Wissink y Meijer, 2004; Estévez, Musitu y Herrero, en prensa; Martínez, Estévez y Jiménez, 2003), factores que fácilmente pueden desarrollar en el adolescente una autopercepción negativa de su ámbito familiar. Respecto del bienestar emocional, se ha comprobado que los adolescentes victimizados suelen presentar más problemas de estrés, ansiedad, depresión y sentimientos de soledad (Guterman et al., 2002; Herrero, Estévez y Musitu, en prensa; Rigby, 2000; Seals y Young, 2003), por lo que su autoestima emocional podría estar seriamente afectada.

\section{OBJETIVO DEL PRESENTE ESTUDIO}

Los trabajos que examinan conjuntamente varias dimensiones de la autoestima en relación con los problemas de agresión y victimización en la escuela son muy escasos en nuestro contexto nacional, especialmente aquellos que consideran las dimensiones familiar y emocional, mientras que los trabajos sobre la violencia escolar son cada vez más abundantes, probablemente porque la violencia escolar es cada vez más un problema de todos. Conocer cómo sienten y cómo se perciben a sí mismos los adolescentes implicados en esta problemática, puede resultar de gran utilidad en el diseño de intervenciones efectivas capaces de responder a estos problemas de convivencia en la escuela (Morales y Costa, 2001). En este sentido, el presente estudio tiene como objetivo analizar las diferencias en autoestima entre adolescentes con problemas de violencia en la escuela, adolescentes victimizados por sus iguales y adolescentes que son al mismo tiempo agresores y víctimas en el contexto escolar. En este trabajo se adopta una concepción multidimensional de la autoestima y se analizan las dimensiones familiar, escolar, social y emocional en los tres grupos señalados -agresores, víctimas y agresores/víctimas- y un cuarto grupo de adolescentes que no presentan -o en el que no se conocen- problemas ni de agresividad ni de victimización en la escuela.

\section{MÉTODO}

\section{Muestra}

Participaron en el estudio un total de 
965 adolescentes de ambos sexos y edades comprendidas entre los 11 y los 16 años. Todos los adolescentes de la muestra cursaban estudios de enseñanza media en el momento de la aplicación de los instrumentos en cuatro centros de enseñanza de la Comunidad Valenciana. A partir de las puntuaciones obtenidas por los participantes en una Escala de Violencia Escolar, se agrupó a los sujetos en las siguientes tres categorias: agresor ( $\mathrm{n}=98$ ), victima ( $\mathrm{n}=280$ ) y agresor/victima $(n=266)$. Estas tres categorias se establecieron considerando las puntuaciones por encima del percentil 75 en las siguientes dimensiones: violencia en la escuela y victimización por los iguales. Además, se estableció una cuarta categoría que agrupó a 321 adolescentes que no presentaron problemas de conducta ni de victimización en la escuela. A esta categoría se le denominó "no implicados".

\section{Instrumentos}

Se utilizaron medidas de conducta violenta y victimización en la escuela, así como de autoestima familiar, social, académica y emocional. En concreto, los instrumentos utilizados fueron:

Escala de Violencia Escolar. Se utilizó una escala compuesta por 19 items (Estévez et al., en prensa) algunos de los cuales se adaptaron de Emler y Reicher (1995). En esta escala, los adolescentes indicaron la frecuencia con que habian participado en 19 comportamientos de carácter violento en la escuela en los últimos 12 meses, en un rango de respuesta de 1 -nunca- a 5 -muchas veces-. La escala mide dos dimensiones: conducta violenta en la escuela (ej. "He agredido o pegado a compañeros del colegio") y victimización (ej. "Alguien del colegio me insultó"). La consistencia interna de estas dimensiones, medida a través del alfa de Cronbach, fue de 0.84 para conducta violenta y 0.82 para victimización.

Escala Multidimensional de Autoestima. Se trata de una escala diseñada, validada y normalizada en España por Musitu, Garcia y Gutiérrez (1994). Esta escala consta de 36 items que evalúan de 1 -nunca- a 5 -siempre- las autopercepciones de los adolescentes en cuatro áreas: la familia (ej. "Mi familia me ayudaria con cualquier tipo de problema"), la escuela (ej. "Soy un/a buen/a estudiante"), el contexto social (ej. "Tengo muchos amigos/as") y las emociones (ej. "Soy un chico/a alegre"). El coeficiente de consistencia interna (Alpha de Cronbach) obtenido para las distintas dimensiones fue de 0.79 para autoestima familiar, 0.86 para autoestima escolar, 0.76 para autoestima social y 0.72 para autoestima emocional.

\section{Procedimiento}

La aplicación de los instrumentos se realizó después de obtener los correspondientes permisos paternos y de la dirección de los centros escolares. Los adolescentes participantes en el estudio cumplimentaron los instrumentos en sus centros de enseñanza durante un periodo regular de clase. La colaboración de profesores y adolescentes fue, en todos los casos, consentida. Asimismo, se insistió a los adolescentes en el anonimato y la confidencialidad de los datos. Varios investigadores previamente entrenados acudieron a los centros de enseñanza para coordinar y revisar la aplicación de los instrumentos.

\section{RESULTADOS}

Para analizar las diferencias entre grupos -agresor, víctima, agresor/víctima y no implicado- se realizó un análi- 
sis de varianza considerando como variable dependiente las cuatro dimensiones de la escala de autoestima: familiar, escolar, social y emocional. Cuando se encontraron diferencias significativas entre grupos se aplicó la prueba de Bonferroni para determinar entre qué grupos concretos se establecían las diferencias en autoestima. En la Tabla 1 se recogen las medias para cada grupo, las desviaciones típicas y los resultados del MANOVA y de la prueba de Bonferroni.

Los resultados del análisis de varianza muestran la existencia de diferencias significativas entre grupos en las cuatro dimensiones de autoestima consideradas. En la dimensión autoestima familiar se encontraron diferencias significativas $\left(F_{\text {familiar, } 5,956}=18.23, \mathrm{p}<.001\right)$ en la siguiente dirección: tanto el grupo de agresores como el de agresores/víctimas presentó una autoestima familiar más baja que el grupo de víctimas y el de adolescentes no implicados. Para el caso de la autoestima escolar las diferencias encontradas fueron en la misma linea $\left(F_{\text {escolar, 5, } 956}=27.12, p<.001\right):$ los dos grupos de adolescentes agresivos -tanto victimizados como no victimizados- informaron de una autoestima escolar más baja, en comparación con las víctimas y el grupo de no implicados. Por tanto, las puntuaciones más elevadas en las dimensiones autoestima familiar y autoestima escolar correspondieron a las víctimas y a aquellos adolescentes que no presentaron problemas de agresión ni de victimización en la escuela.

Respecto de la autoestima social ( $\mathrm{F}_{\text {social }}$ $5,956=7,30, p<.001$ ), el grupo de agresores mostró las puntuaciones más elevadas en esta dimensión, seguido del grupo de agresores/víctimas y de adolescentes no implicados -estos dos grupos no presentaron diferencias significativas entre sí en su percepción de autoestima social- y, finalmente, el de las victimas. Por último, los resultados señalaron diferencias significativas en la dimensión autoestima emocional ( $\mathrm{F}_{\text {emocional, }}$ 5, $956=$ $10.60, \mathrm{p}<.001$ ) entre el grupo de adolescentes agresivos y los otros tres grupos. En este caso, los agresores obtuvieron las puntuaciones más elevadas en autoestima emocional en comparación con las víctimas, los agresores/víctimas y los adolescentes no implicados.

Tabla 1. Medias, desviaciones típicas, resultados del MANOVA y de la prueba Bonferroni

\begin{tabular}{|c|c|c|c|c|c|}
\hline & $\Lambda$ gresor & Víctima & $\Lambda$ gresor/Víctima & No implicado & $\mathrm{l}_{5,956}$ \\
\hline 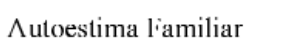 & $24.96(3.94)^{b}$ & $26.03(3.61)^{\mathrm{a}}$ & $24.55(4.37)^{b}$ & $26.74(3.52)^{\mathrm{a}}$ & $18.23^{* * *}$ \\
\hline Autocstima Escolar & $17.84(4.80)^{\mathrm{b}}$ & $21.28(4.43)^{\mathrm{a}}$ & $18.82(4.65)^{\mathrm{b}}$ & $21.53(4.56)^{\mathrm{a}}$ & $27.12^{* * *}$ \\
\hline 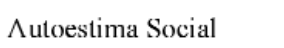 & $25.43(3.53)^{\mathrm{a}}$ & $23.33(3.67)^{\mathrm{c}}$ & $24.31(3.81)^{b}$ & $24.14(3.88)^{b}$ & $7.500^{* * *}$ \\
\hline 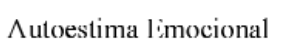 & $13.83(2.92)^{\mathrm{a}}$ & $12.26(3.34)^{b}$ & $12.34(3.33)^{\mathrm{b}}$ & $12.60(3.33)^{b}$ & $10.60^{* * *}$ \\
\hline
\end{tabular}

Prueba de Bonferroni/ Bonferroni correction: $\alpha=0.05 ; \mathrm{a}>\mathrm{b}>\mathrm{c}$

${ }^{* * *} p<.001$ 
En resumen, los resultados obtenidos vienen a indicar que el grupo de agresores/victimas es el grupo que, en general, presenta las puntuaciones más bajas en las cuatro dimensiones de autoestima analizadas. Sólo el grupo de víctimas obtuvo una valoración más negativa en una de las dimensiones: en autoestima social. Por otro lado, agresores, victimas y adolescentes no implicados, se valoran a sí mismos más positivamente en algunos aspectos específicos de la autoestima que en otros: los agresores en los dominios social y emocional, y las víctimas y los adolescentes no implicados en los dominios familiar y escolar.

\section{DISCUSIÓN Y CONCLUSIONES}

En el presente estudio se han analizado las diferencias entre agresores y víctimas en autoestima familiar, escolar, social y emocional. Los resultados indican que, en general, los adolescentes victimizados en la escuela se perciben a sí mismos más negativamente en los dominios social y emocional de la autoestima, en comparación con los agresores. En este sentido, investigaciones previas señalan que las víctimas de violencia escolar suelen presentar desórdenes emocionales como estrés, ansiedad y sintomas depresivos (Juvonen, Nishina y Graham, 2000; Kaltiala-Heino, Rimpela, Rantanen y Rimpela, 2000; Seals y Young, 2003), así como problemas de aislamiento, exclusión social en la escuela y dificultades para integrarse socialmente en el grupo de compañeros (Prinstein, Boergers y Vernberg, 2001; Storch y Masia-Warner, 2004). Las víctimas son percibidas por sus compañeros como personas tristes, inseguras y timidas que tienen pocos o ningún amigo (Díaz-Aguado, 2002; Trianes, 2000). Los resultados obtenidos en la presente investigación sugieren que esa imagen de las víctimas como personas tristes, deprimidas y solas es, de hecho, compartida por las propias victimas.

Sin embargo, las autopercepciones de las víctimas relativas a los contextos familiar y escolar, son más positivas que en el caso de ambos grupos de agresores, e incluso tan positivas como las autopercepciones de los adolescentes sin problemas de agresión o victimización en la escuela. Así, aunque las víctimas sostienen que en ocasiones se sienten sobreprotegidas por sus padres (Cerezo, 1999), en general, se muestran satisfechas con su entorno familiar, mientras que por el contrario, los agresores suelen informar de la existencia de relaciones hostiles y desafiantes con sus padres (Dekovic et al., 2004; Kazdin, 1995). Respecto del contexto escolar, estudios previos han constatado que las víctimas presentan un buen rendimiento académico y no muestran más dificultades académicas que el resto de compañeros (Olweus, 1998), mientras que los agresores suelen ser repetidores con un bajo rendimiento académico y muestran normalmente actitudes desfavorables hacia la escuela, el profesorado y los estudios (Cerezo, 1999; Estévez, Linares, Cava y Martinez, 2002).

Los adolescentes agresivos, sin embargo, se perciben a sí mismos como altamente competentes en los dominios social y emocional, más competentes incluso que sus compañeros no implicados en problemas de agresión o victimización en la escuela. Este resultado va en la linea del apuntado por Angold y Costello (1993), quienes no encontraron apenas co-ocurrencia entre el comportamiento agresivo en adolescentes y la existencia de problemas emocionales. Así, por ejemplo, se ha constatado que la agresividad y la sintomatología depresiva co-ocurren únicamente en el 5-8\% de los adolescentes (Garnefski y Diekstra, 1997; Ge, Best, Conger y Simons, 
1996), lo que descarta que el comportamiento agresivo sea una fuente directa de estrés y ansiedad en la adolescencia (Herrero et al., en prensa). Muy al contrario, los datos reflejan que la mayoría de los agresores tienen una alta autoestima emocional (Brendgen, Vitaro, Turgeon, Poulin y Wanner, 2004). Una posible explicación es que los adolescentes agresivos son en numerosas ocasiones figuras importantes en su grupo de iguales (Gilford-Smith y Brownell, 2003; Hawley y Vaughn, 2003) e incluso populares y queridos entre sus compañeros (Salmivalli, 1998), lo que les hace disfrutar de ciertos beneficios sociales que se reflejan en su bienestar tanto emocional como social (Little, Brauner, Jones, Nock y Hawley, 2003).

Finalmente, el grupo de agresores/ victimas es el que presenta un cuadro más negativo de autopercepciones. Comparte características tanto con los agresores como con las víctimas, pero refleja el lado más negativo de cada uno de ellos. Así, por un lado, los agresores/víctimas se valoran más negativamente que los agresores en las dimensiones social y emocional de la autoestima. Estos resultados coinciden con los apuntados por Austin y Joseph (1996), quienes también encontraron en su estudio que el grupo de agresores y el de agresores victimiza- dos diferían entre sí fundamentalmente en los dominios social y emocional. Por otro lado, los agresores/víctimas se valoran más negativamente que las víctimas en los dominios familiar y social de la autoestima.

Para concluir, los resultados de esta investigación señalan la importancia de adoptar una perspectiva multidimensional en el estudio de la autoestima en agresores y víctimas en la escuela, así como la necesidad de considerar las dimensiones familiar y escolar en este análisis, puesto que ambas dimensiones han presentado claras diferencias entre los grupos de estudiantes considerados. No obstante, la interpretación de estos resultados debe realizarse con cautela debido a la naturaleza correlacional de los datos. En este sentido, sería interesante incorporar la dimensión temporal en futuras investigaciones para poder analizar la estabilidad de las relaciones establecidas. Aún así, desde el punto de vista exploratorio, los resultados aquí presentados pueden ser de utilidad para orientar el diseño de programas de intervención destinados a modificar el comportamiento desviado de algunos estudiantes, mejorar la calidad de vida de las víctimas de violencia escolar, así como fomentar la convivencia pacífica en los centros de enseñanza. 


\section{BIBLIOGRAFÍA}

Andreou, E. (2000). Bully/Victim problems and their association with psychological constructs in 8-to 12-year old Greek schoolchildren. Aggressive Behavior, 26, 49-56.

Angold, A. y Costello, E. J. (1993). Depressive comorbidity in children and adolescentes: Empiri$\mathrm{cal}$, theoretical and methodological sigues. American Journal of Psychiatry, 150, 1779-1791.

Austin, S. y Joseph, S. (1996). Assessment of bully/victim problems in 8 to 11 years old. British Journal of Educational Psychology, 66, 447-456.

Brendgen, M., Vitaro, F., Turgeon, L., Poulin, F. y Wanner, B. (2004). Is there a dark side of positive illusions? Overestimation of social competence and subsequent adjustment in aggressive and nonaggressive children. Journal of Abnormal Child Psychology, 32, 305-320.

Cava. M. J. y Musitu, G. (2003). La potenciación de la autoestima en la escuela. Barcelona: Paidós.

Cava, M. J., Musitu, G. y Vera, A. (2000). Efectos directos e indirectos de la autoestima en el ánimo depresivo. Revista Mexicana de Psicología, $17,151-161$.

Cerezo, F. (1999). Conductas agresivas en la edad escolar. Madrid: Pirámide.

Dekovic, M., Wissink, I. B. y Meijer, A. M. (2004). The role of family and peer relations in adolescent antisocial behaviour: comparison of four ethnic groups. Journal of Adolescence, 27, 497-514.

Díaz-Aguado, M. J. (2002). Convivencia escolar y prevención de la violencia. Madrid: Publicaciones del Ministerio de Educación y Ciencia, CNICE.

Dorothy, S. y Jerry, Y. (2003). Bullying and victimization: prevalence and relationship to gender, grade level, ethnicity, self-esteem, and depression. Adolescence, 38, 735-747.

Emler, N. y Reicher, S. (1995). Adolescence and Delinquency. Oxford: Blackwell.

Estévez, E., Llinares, L., Cava, M.J., y Martínez, B. (2002). Conducta disruptiva y actitud hacia la autoridad institucional en adolescentes: el rol de la escuela. Actas del IV Congreso Estatal de Escuelas de Trabajo Social. Alicante.
Estévez, E., Musitu, G. y Herrero, J. (2005). The influence of violent behavior and victimization at school on psychological distress: the role of parents and teachers. Adolescence, 40, 183-196.

Garnefski, N. y Diekstra, R. F. W. (1997). Comorbidity of behavioral, emocional, and congnitive problems in adolescente. Journal of Youth and Adolescence, 26, 321-338.

Ge, X., Best, K. M., Conger, R. M. y Simons, R. L. (1996). Parenting behaviors and the occurrence and co-occurrence of adolescent depressive symptoms and conduct problems. Developmental Psychology, 32, 717-731.

Gifford-Smith, M. E. y Brownell, C. A. (2003). Childhood peer relationships: social acceptance, friendships, and social network. Journal of School Psychology, 41, 235-284.

Guterman, N. B., Hahm, H. C. y Cameron, M. (2002). Adolescent victimization and subsequent use of mental health counselling services. Journal of adolescent Health, 30, 336-345.

Hawley, P. y Vaughn, B. (2003). Aggression and adaptation: The bright side to bad behavior. Introduction to special volume. Merrill-Palmer Quarterly, 49, 239-244.

Herrero, J., Estévez, E. Musitu, G. (2003). Deviant behaviour and victimization at school: exploring the role of parents and teachers in adolescent psychological distress. Journal of Adolescence, disponible online.

Herrero, J., Musitu, G. y Gracia, E. (1995). Autoestima y depresión: relaciones directas versus indirectas. Revista de Psicología Social, 10, 191-204.

Juvonen, J., Nishina, A. y Graham, S. (2000). Peer harassment, psychological adjustment, and school functioning in early adolescence. Journal of Educational Psychology, 92, 349-359.

Kaltiala-Heino, R., Rimpelä, M., Rantanen, P. y Rimpelä, A. (2000). Bullying at school: An indicator of adolescents at risk for mental disorders. Journal of Adolescence, 23, 671-674.

Kazdin, A. E. (1995). Conduct disorders in childhood and adolescence. London: Sage Publications.

Little, T., Brauner, J., Jones, S., Nock, M. y 
Hawley, P. (2003). Rethinking aggression: A typological examination of the functions of aggression. Merrill-Palmer Quarterly, 49, 343-369.

Martinez, B., Estévez, E. y Jiménez, T. (2003) Influencia del funcionamiento familiar en la conducta disruptiva en la adolescencia. Encuentros en Psicología Social, 1, 64-67.

Morales, J. M. y Costa, M. C. (2001). La prevención de la violencia en la infancia y la adolescencia. Una aproximación conceptual integral. Intervención Psicosocial, 10 (2), 221-239.

Musitu, G., Buelga, S., Lila, M. S. y Cava, M. J. (2001). Familia y Adolescencia. Madrid: Sintesis.

Musitu, G., García, J. F. y Gutiérrez, M. (1994). AFA-Autoconcepto Forma A ( $2^{\underline{a}}$ edición). Madrid: TEA.

Mynard, H y Joseph, S. (1997). Bully/Victim problema and their association with Eysenck's personality dimensions in 8 to 13 years-olds. British Journal of Educational Psychology, 67 (1), 51-54.

Olweus, D. (1998). Conductas de acoso y amenaza entre escolares. Madrid: Morata.

O'Moore, A.M. (1997). What do teachers need to know? En M. Elliot (Ed.), Bullying: A practical guide to coping for schools (pp. 151-166). Londres: Pitman/Kidscape.

O'Moore, M. y Kirkham, C. (2001). Self-esteem and its relationship to bullying behavior. Aggressive behavior, 27, 269-283.

Prinstein, M. J., Boergers, J. y Vernberg, E. M. (2001). Overt and relational aggression in adoles- cents : social-psychological adjustment of aggressors and victims. Journal of Clinical Child Psychology, 30, 479-491

Rigby, K. (2000). Effects of peer victimization in schools and perceived social support on adolescent well-being. Journal of Adolescence, 23, 57-68.

Rigby, K. y Slee, P. (1992). Bullying among Australian school children: reported behavior and attitudes toward victims. Journal of School Psychology, $131,615-627$

Rosenberg, M. (1986). Conceiving the Self. Malabar, FL: Krieger.

Rosenberg, M., Schooler, C., Schoenbach, C. y Rosenberg, F. (1995). Global self-esteem and specific self-esteem: different concepts, different outcomes. American Sociological Review, 60, 141-156.

Salmivalli, C. (1998). Intelligent, attractive, well-behaving, unhappy: the structure of adolescents' self-concept and its relations to their social behavior. Journal of Research on Adolescence, 8, 333-354.

Seals, D. y Young, J. (2003). Bullying and victimization: prevalence and relationship to gender, grade level, ethnicity, self-esteem, and depression. Adolescence, 38, 735-747.

Storch, E. A. y Masia-Warner, C. (2004). The relationship of peer victimization to social anxiety and loneliness in adolescent females. Journal of Adolescence, 27, 351-362.

Trianes, M. V. (2000). La violencia en contextos escolares. Málaga: Aljibe. 